International Journal of Psychological Research and Reviews (ISSN:2639-6041)

\title{
Spirituality and Meditation During a COVID-19 Lockdown
}

\author{
Tiffany Field ${ }^{1,2}$, Samantha Poling ${ }^{2}$, Shantay Mines ${ }^{2}$, Debra Bendell ${ }^{2}$, Connie Veazey ${ }^{2}$ \\ ${ }^{1}$ University of Miami/Miller School of Medicine \\ ${ }^{2}$ Fielding Graduate University
}

ABSTRACT

Spirituality and meditation have been associated with emotional and physical well-being, but studies on their relationships are

${ }^{*}$ Correspondence to Author:

surprisingly missing from the COVID-19 lockdown literature. In Tiffany Field ${ }^{1,2}$,

this Survey Monkey study conducted during a COVID-19 lock${ }^{1}$ University of Miami/Miller School down, $72 \%$ of 260 respondents reported feeling spiritual and of Medicine; ${ }^{2}$ Fielding Graduate $48 \%$ meditated. Correlation analyses suggested that spirituality and meditation were positively correlated. Spirituality was also positively related to health practices and working on projects and negatively correlated with COVID-related stress, anxiety, depression, fatigue, and sleep disturbances. And, meditation was positively correlated with health and working scale scores. SpiriUniversity

tuality and meditation may be buffers for these COVID-19 related stressors.

How to cite this article:

Tiffany Field, Samantha Poling, Shantay Mines, Debra Bendell, Connie Veazey.Spirituality and Meditation During a COVID-19 Lockdown. International Journal of Psychological Research and Re-

Keywords; Spirituality, Meditation, COVID-19 Lockdown, Stress, views, 2021, 4:53.

Depression; Spirituality and Meditation During a COVID-19 Lockdown

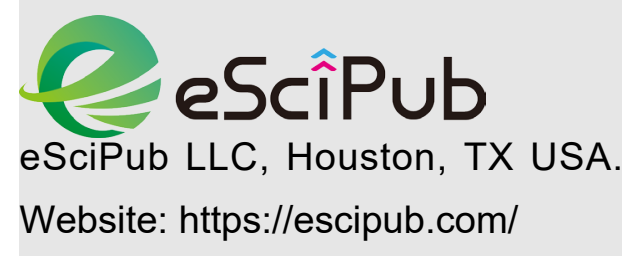


The vast COVID-19 literature has derived from research conducted in many parts of the world. A recent literature search on the term COVID-19 yielded 3,218 published papers in 2020 to date. However, only 204 papers could be found when COVID-19 was combined with the term health and fewer papers when paired with mental health (43 studies) and even more rarely when mental health was specified as anxiety (18 papers), depression (6) or sleep disturbances (3). In addition, except for exercise (3 papers), no studies were found on potential buffers for negative mood states and sleep disturbances. One exception was a correlation analysis on this database suggesting that stress was positively related to feeling touch deprived but negatively related to feeling spiritual and meditating, highlighting spirituality and meditating as potential buffers for lockdown stress (Field, et al, 2020a).

Spirituality has been defined as "standing outside of time and space" (Laird et al., 2017) and it could be said that meditation facilitates that process (Carmody et al., 2008). That experience might make spirituality and meditation therapeutic during lockdowns. Spirituality has been most frequently coupled with religiosity when studied in the context of health, although the focus of many studies has been an attempt to differentiate the health effects of spirituality and religiosity (Rosmarin et al., 2000; Seeman et al., 2003). Meditation has been most recently associated with mindfulness meditation, although other forms like transcendental meditation preceded mindfulness meditation (Seeman et al., 2003). A neuroimaging study has suggested that these different forms have equivalent effects (Brandmyer et al., 2018), while others suggest that the different forms of meditation result in different neural oscillatory activity (Lee et al., 2017).

Although literature on spirituality and meditation during COVID-19 is non-existent, both have been considered healthful activities, especially in reducing mental health conditions. The evidence-based research on the associations between these and physical and mental health includes the reduction of stress, depression, anxiety, sleep disturbances and PTSD symptoms (Braan \& Koenig, 2019; GonzalezValero et al., 2019; Hilton et al., 2017; Knowlden et al., 2018; Saeed et al., 2019; Thomas et al., 2017; Tsai et al., 2019; Wahleh, 2018; Yadav et al., 2017; Yang et al., 2018). For example, in a recent review, spirituality reputedly reduced depression in $49 \%$ of 152 studies (Braan \& Koenig, 2019), and spirituality was negatively related to sleep disturbances in a crosssectional stratified randomized sample (Tsai et al., 2019) as well as a MANOVA (Knowlden et al., 2018). The spirituality relationship with sleep disturbances may be mediated by the reduction of stress (Yadav et al., 2017).

The recent literature on meditation effects has been more expansive than that of spirituality effects. These include the decrease of depression (Saeed et al., 2019), comorbid depression and anxiety (Thomas et al., 2017) and depression, anxiety, and stress in a metaanalysis on 34 studies (Gonzalez-Valero et al., 2019). And the effects of meditation on these mood states have been noted to persist as long as one month following a 30-day meditation intervention Yang et al., 2018). Meditation has also reduced insomnia (Wahleh, 2018). And, in a meta-analysis on ten trials, meditation reduced PTSD symptoms (Hilton et al., 2017).

The purpose of the present data analyses was to assess the relationships between feeling spiritual and meditating and COVID-related stress, anxiety, depression, fatigue, sleep disturbances, and PTSD symptoms in individuals experiencing a COVID-19 lockdown. Feeling spiritual and meditating were expected to be associated with less COVID-related stress, anxiety. depression, fatigue, sleep disturbances and PTSD symptoms. In addition, they were expected to be related to other positive activities including working on projects, social media/communication, and connecting with others. 


\section{Methods}

\section{Participants}

A G* power analysis indicated that a sample size of 224 was required for an alpha of .05 and $80 \%$ power. The participants included 260 individuals who ranged in age from 18-82 ( $M=47$ years). Gender was distributed $79 \%$ female, $18 \%$ male and $3 \%$ other (non-specified). Ethnicity was distributed 68\% Non-Hispanic White, 21\% Hispanic, 3\% Black and 8\% other (nonspecified). Professions were distributed 35\% office worker, 30\% academic, 15\% managerial, $12 \%$ medical and $8 \%$ labor. The average income was \$72,572, 28\% were unemployed and $69 \%$ worked at home. Twenty-three per cent lived alone.

\section{Procedure}

A flyer was posted on Facebook giving a brief description of the study including some sample items and the age criterion being greater than 18 years. The Facebook flyer included a link to the survey on Survey Monkey which included 11 scales for a total of 87 items. The survey was four weeks duration (April 1-30, 2020), and the data were directly transported to SPSS for data analyses.

\section{Measures}

The survey included several demographic items including those already mentioned (age, gender, ethnicity, profession, income, type of employment, working at home, and living alone). The following five scales were created specifically for this survey to relate to activities and stress associated with the COVID-19 lockdown (Field et al., 2020b). The participants rated the items on the scales from zero meaning "not at all" to three meaning "a lot" including the:

1) Health Scale (15 items) (Cronbach's alpha=.66) which included exercise (inside exercise, outside exercise and outside exercise with others as well as the types of exercise), touching (touching partner, kids and self as well as the types of touching), COVID- 19-related safety practices including washing hands and social distancing, selfcare, feeling spiritual, meditating, and liking being at home. A principal components analysis (rotation method varimax with Kaiser normalization) yielded three components contributing to $47 \%$ of the variance on the Health Scale score (see table 1 for the components, items, their loadings and variance explained).

2) Media/Communications Scale (10 items) (Cronbach's alpha=.58) including talking on the phone, texting, on Internet, gaming, on Facebook/Instagram, spending time receiving and sending messages/media about the virus, engaging in Zoom/Skype/Facetime activities (e.g. Yoga, meditation), watching the news, watching other TV programs, and watching movies. A principal components analysis (rotation method varimax with Kaiser normalization) yielded four components contributing to $61 \%$ of the variance on the Media/Communication Scale score (see table 2 for the components, items, loadings and variance explained).

Table 1. Health Scale components, items, loadings and variance explained.

\begin{tabular}{llc}
\hline Items & Loadings & Variance explained \\
\hline Component 1:Spirituality/self-care & .77 & \\
$\quad$ Feeling Spiritual & .74 & \\
Meditating & .68 & $23 \%$ \\
$\quad$ Self-Care & .75 & \\
Component 2: Touching & .72 & $14 \%$ \\
$\quad$ Touching your kids & & \\
$\quad$ Touching your partner/friend & -.89 & $10 \%$ \\
Component 3: Exercise & -.76 & \\
$\quad$ Outdoor exercise & & \\
$\quad$ Exercise with someone else & \multicolumn{2}{l}{ IJPRR:https://escipub.com/international-journal-of-psychological-research-and-reviews/ }
\end{tabular}


Table 2. Media/Communications Scale components, items, loadings and variance explained.

\begin{tabular}{llc}
\hline Items & Loadings & Variance explained \\
\hline Component 1:Entertainment & .84 & \\
Watching movies & .80 & $23 \%$ \\
Watching tv programs & .80 & \\
Component 2:Communication & .70 & \\
$\quad$ Phone use & .63 & $14 \%$ \\
Texting & & \\
Zoom & .78 & $13 \%$ \\
Component 3:Social media & .60 & \\
Internet & & $11 \%$ \\
$\quad$ Facebook time & .79 & \\
Component 4: COVID-19 News & .60 & \\
Watching the news & & \\
Messaging about the virus &
\end{tabular}

Table 3. Stress scale components, items, loadings and variance explained.

\begin{tabular}{lll}
\hline Items & Loadings & Variance explained \\
\hline Component 1: Stimulation deprivation & & \\
Feeling isolated & .86 & \\
Feeling lonely & .86 & \\
Feeling bored & .74 & \\
Getting “cabin fever" & .70 & $34 \%$ \\
Feeling touch deprived & .65 & \\
Component 2:Worrying & & \\
Worried about finances & .67 & \\
Worried about the virus & .47 & \\
Component 3:Stress behaviors & & $12 \%$ \\
Napping & .68 & \\
Snacking & .53 & \\
\hline
\end{tabular}

3) Connecting Scale (4 items) (Cronbach's alpha $=.41$ ) which included connecting with friends, trying to connect with old friends, helping children do homework, and receiving support from others;

4) Working Scale (6 items) (Cronbach's alpha $=.61$ ) including cooking, caregiving, housekeeping, paperwork, creative work, and working on projects/hobbies; and
5) Stress Scale (11 items) (Cronbach's alpha=.78) which included worrying about getting the virus, worrying about your financial status, wanting this experience to end, feeling isolated, feeling lonely, feeling bored, feeling touch deprived, snacking, drinking alcohol, napping, and getting "cabin fever". . A principal components analysis (rotation method varimax with Kaiser normalization) yielded three components contributing to $56 \%$ of the variance on the Media/Communication Scale score (see 
table 3 for the components, items, loadings and variance explained).

The standardized scales on the survey included 4 PROMIS Subscales (Dewitt et al., 2018) (each item was rated on a 5 -point scale as $1=$ never, $2=$ rarely, $3=$ sometimes, $4=$ often, and $5=a l w a y s)$ which included the:

1) PROMIS Anxiety Subscale (4 items) (Cronbach's alpha $=.88$ ) on the items I felt fearful, I found it hard to focus on anything other than my anxiety, my worries overwhelmed me, and I felt uneasy;

2) PROMIS Depression Subscale (4 items) (Cronbach's alpha=.91) including I felt worthless, helpless, depressed, and hopeless;

3) PROMIS Fatigue Subscale (3 items) (Cronbach's alpha=.92) including I felt fatigued, I had trouble starting things because I'm tired, and I felt run-down; and

4) PROMIS Sleep Disturbance Subscale (4 items) (Cronbach's alpha $=.86$ ) which included my sleep quality was bad, my sleep is not refreshing, I had a problem with my sleep, and I had difficulty falling asleep.

The second standardized scale was a PTSD Screener entitled "PTSD-8: A short PTSD Inventory" (8 items) (Cronbach's alpha=.92) (Hansen et al., 2010). This inventory is introduced by the statement "If you're being reminded of a traumatic experience, please rate how much the following have bothered you during the lockdown" as: 0) not at all, 1) rarely, 2) sometimes, and 3) most of the time. The items are: recurrent thoughts and memories of the event, feeling as though the event is happening again, recurrent nightmares about the event, sudden emotional or physical reactions when reminded of the event, avoiding activities that remind you of the event, avoiding thoughts or feelings associated with the event, feeling jumpy/easily startled, and feeling on guard.

\section{Results}

\section{Correlation Analyses Yielding Significant Coefficients for Feeling Spiritual}

The Survey Monkey output indicated that $72 \%$ of the sample reported feeling spiritual (rated 0
(28\%) none, 1 (28\%), 2 (25\%), $3(19 \%)$ a lot). Correlation analyses revealed a number of significant coefficients for feeling spiritual (at the $p<.05$ level with most at the $p=.0001$ level) including the correlation between feeling spiritual and meditating $(r=.46, p=.001)$ and the following (see Table 4 for the correlation coefficients for the scales' total scores):1) a positive correlation for the Health Scale total score, and the items including more indoor exercise, more exercise with someone else, more self-touch, more liking being home, and more self-care; 2) both positive and negative correlations for items on the Media/Communication Scale indicating that spirituality was related to more phoning, less internet and less messaging about the virus; 3 ) a positive correlation with the total score on the Connecting Scale and on the items connecting with friends and trying to connect with old friends; 4) a positive correlation with the Working Scale for the total score and the items including more cooking, more caregiving, more housekeeping, more creative work and more working on projects/hobbies; 5) a negative correlation with the total score on the Stress Scale and for its items indicating less feeling isolated, less lonely, less bored, less touch deprived, and less cabin fever; 6) a negative correlation for the PROMIS Anxiety Subscale total score and its items indicating less focus on anxiety, overwhelming worries and feeling uneasy; 7) a negative correlation for the total score on the PROMIS Depression Subscale, and all its items including less feeling worthless, helpless, depressed, and hopeless; 8) a negative correlation for the total score on the PROMIS Fatigue Subscale, and all its items indicating less fatigue, tired, and run-down; 9) a negative correlation for the total score on the PROMIS Sleep Disturbance Subscale, and all its items including quality of sleep, refreshing sleep, problems with sleep, and falling asleep; and 10) a negative correlation for the total score on the PTSD-8 Inventory and its item feeling the event is happening again. 
Table 4. Correlation coefficients for significant relationships between feeling spiritual ratings and scores on COVID-19 Lockdown Activities Survey scales and subscales.

\begin{tabular}{lcl}
\hline Measure & Correlation coefficient & p level \\
\hline Health Scale Score & .55 & .0001 \\
Connecting Scale Score & .18 & .005 \\
Working Scale Score & .25 & .0001 \\
Stress Scale Score & -.25 & .02 \\
PROMIS Anxiety Subscale Score & -.17 & .008 \\
PROMIS Depression Subscale Score & -.24 & .0001 \\
PROMIS Fatigue Subscale Score & -.23 & .0001 \\
PROMIS Sleep Disturbance Subscale Score & -.20 & .001 \\
Posttraumatic Stress-8 Inventory Score & -.14 & .05 \\
\hline
\end{tabular}

Table 5. Correlation coefficients for significant relationships between meditating ratings and scores on COVID-19 Lockdown Activities Survey scales and subscales.

\begin{tabular}{lcl}
\hline Measure & Correlation coefficient & p level \\
\hline Health Scale Score & .53 & .0001 \\
Working Scale Score & .23 & .0001 \\
PROMIS Sleep Disturbance Subscale Score & -.12 & .05 \\
\hline
\end{tabular}

Correlation Analyses Yielding Significant Coefficients for Meditating

The Survey Monkey output indicated that $48 \%$ of the sample reported meditating (rated $0(52 \%)$ none, 1 (23\%), 2 (13\%), 3 (12\%) a lot). Correlation analyses revealed a number of significant coefficients for meditating (at the $\mathrm{p}<.05$ level) including the correlation between feeling spiritual and meditating (.46) and the following (see Table 5 for the correlation coefficients for the scales' total scores):1) a positive correlation for the Health Scale total score, and the items including more indoor exercise, more self-touch, more social distancing, more liking being at home, and more self-care; 2) a positive correlation on the Media/Communication Scale indicating more zooming;3) a positive correlation with the Connecting Scale suggesting more connecting with old friends; 4)a positive correlation with the Working Scale for the total score and its items indicating more housekeeping, more paperwork, more creative work and more working on projects/hobbies; 5) a negative correlation with the Stress Scale items including feeling less bored and less snacking; 6) a negative correlation for the depression item on the PROMIS Depression Subscale; and 7) a negative correlation for the PROMIS Sleep Disturbance Subscale total score and the quality of sleep item.

\section{Analyses of Variance (ANOVAs) on Feeling Spiritual Versus Not Feeling Spiritual Groups} ANOVAs were conducted to provide confirmatory data for the correlation analyses. The sample was divided into a feeling spiritual group which was comprised of those respondents with 1-3 scores (a little to a lot) on the feeling spiritual rating and a non-feeling spiritual group that included participants who scored 0 (not at all) on that rating. The ANOVAs comparing the feeling spiritual versus the not feeling spiritual group provided confirmatory data for the scales and subscales that were significantly correlated with the spirituality variable. The ANOVAs can be seen in Table 6 . The exceptions were that both the Connecting and the Working Scale scores were positively ournal-of-psychological-research-and-reviews/ 6 
correlated with the spirituality variable, but the spirituality and non-spirituality groups did not significantly differ on those two scales. And, the Media Scale scores were not significantly correlated with the spirituality variable, but the spirituality and non-spirituality groups significantly differed on that total scale score. The MANOVA for this set of ANOVAs was significant (Wilks' Lambda $\mathrm{F}=7.89, p=.0001$, eta ${ }^{2}$ $=.19)$.

Table 6. Mean scale scores for significant ANOVAs for feeling spiritual versus not feeling spiritual groups (standard deviations in parentheses).

\begin{tabular}{lccccc}
\hline Measure & Non-spiritual & \multicolumn{1}{c}{ Spiritual } & F value & p level & eta $^{2}$ \\
\hline Health & $31.37(5.47)$ & $33.42(5.31)$ & 4.93 & .03 & .02 \\
Media & $27.17(3.99)$ & $25.43(5.09)$ & 6.54 & .01 & .03 \\
Stress & $28.92(5.55)$ & $19.69(3.03)$ & 124.14 & .0001 & .33 \\
Anxiety & $10.54(3.43)$ & $6.98(2.60)$ & 45.57 & .0001 & .15 \\
Depression & $9.08(3.91)$ & $5.33(2.22)$ & 41.89 & .0001 & .14 \\
Fatigue & $8.63(2.99)$ & $5.69(2.48)$ & 40.64 & .0001 & .14 \\
Sleep Disturbance & $14.42(4.34)$ & $11.28(4.59)$ & 20.64 & .001 & .08 \\
PTSD & $15.24(5.85)$ & $11.58(4.87)$ & 11.22 & .001 & .06 \\
\hline
\end{tabular}

Table 7. Mean scale scores for significant ANOVAs for meditating and not meditating groups (standard deviations in parentheses).

\begin{tabular}{llllll}
\hline Measure & Non-meditating & Meditating & F value & p level & eta $^{2}$ \\
\hline Health Scale Score & $29.44(4.73)$ & $34.44(5.08)$ & 58.15 & .0001 & .21 \\
Working Scale Score & $14.89(3.55)$ & $16.13(3.34)$ & 8.27 & .004 & .03 \\
\hline
\end{tabular}

\section{Analyses of Variance (ANOVAs) on Meditating Versus Not Meditating Groups}

Similarly, a meditating group was comprised of those respondents with 1-3 scores (a little to a lot) on the meditating rating and a nonmeditating group that included participants who scored 0 (not at all) on that rating. The ANOVAs comparing the meditating versus the not meditating group provided confirmatory data for the scales and subscales that were significantly correlated with the meditating variable. The ANOVAs can be seen in Table 7. The only exception was that although the Sleep Disturbance Subscale scores were positively correlated with the meditating variable, the meditating versus non-meditating groups did not significantly differ on that subscale. The MANOVA for this set of ANOVAs was significant (Wilks' Lambda $\mathrm{F}=28.23, p=.0001$, eta ${ }^{2}=.21$ ).

\section{Discussion}

As might be expected, spirituality and meditation were significantly correlated. As has been suggested, spirituality is "an individual's ability to stand outside an immediate sense of time and place to view life from a larger, more objective perspective" (Laird et al., 2017). And it could be said that meditation facilitates that process (Carmody et al., 2008). Surprisingly, however, while $72 \%$ of the respondents reported feeling spiritual, only $48 \%$ reported engaging in meditation. Nonetheless, neither spirituality nor meditation were related to being worried about the virus or about finances which would be consistent with "standing outside an immediate sense of time and place" (Laird et al., 2017). And, both spirituality and meditation were related to connecting with friends and engaging in various kinds of work including creative activities and hobbies and liking being at home IJPRR:https://escipub.com/international-journal-of-psychological-research-and-reviews/ 
during the lockdown which would be consistent with their definitions.

The sleep disturbance scale scores were also negatively related to both the spirituality and meditation variables which is consistent with the pre-COVID literature (Tsai et al., 2019; Knowlden et al., 2018) and with at least one COVID-19 paper on sleep disturbances (Field et al., 2020a). And, meditation has been noted to reduce insomnia (Wahleh, 2018). The reduction in sleep disturbances may have, in turn, mediated the relationships between spirituality, meditation, and health.

The specific health variables that related to both spirituality and meditation included indoor exercise (the most frequent being yoga which includes meditation), self-touch (again, yoga as it was given as the most frequent example in the item on self-touch), and self-care. Both exercise and touch have been buffers for COVID-19 stress in previous research (Field et al., 2020b). Although self-care was not operationally defined, the respondents may have interpreted self-care as caring for their health. Much of the literature on both spirituality and meditation has focused on their relationships to health (Rosmarin et al., 2000; Seeman et al., 2003). One of the potential underlying mechanisms that has been proposed for the meditation-health relationship is that meditation reduces stress and, in turn, suppresses chronic inflammation (Anderson et al., 2017).

Surprisingly, spirituality but not meditation was negatively related to depression. The absence of a relationship between meditation and depression was inconsistent with the literature reviewed above (Saeed et al., 2019; Thomas et al., 2017). This may relate to the lesser opportunity for group meditation during COVID19 lockdowns. The negative relationship between spirituality and depression was consistent with pre-COVID literature (Braan \& Koenig, 2019). As already mentioned, spirituality was related to reduced depression in $49 \%$ of 152 studies in a recent review on the spirituality literature (Braan \& Koenig, 2019). Different potential underlying mechanisms have been suggested for the spirituality-depression relationship including increased dopamine, serotonin, and oxytocin genes associated with both spirituality and the absence of depression (Anderson et al., 2017). And spirituality has been positively related and depression negatively related to alpha EEG waves (Panier et al., 2020). Further, in a meta-analysis on physiological markers of health, decreased blood pressure and C-reactive protein were frequently noted (Shattuck \& Muehlenbein, 2020). These, in turn, could contribute to the health effects by the associated reduction in stress and inflammation (Anderson et al., 2017).

Spirituality was negatively related to the stress scale total scores and its items, and the spiritual and the non-spiritual groups differed on the stress variables. The negative relationship between spirituality and stress was unexpected inasmuch as research on the stress scale variables could not be found in the pre-COVID literature on spirituality. Several individual variables contributed to the stress score including feeling isolated, lonely, bored, touch deprived, and having cabin fever. That loneliness was one of the significant stress items was not surprising given that loneliness was previously reported as the most robust factor in a factor analysis on the stress scale that was designed specifically to tap COVID-related stress (Field et al., 2020a). These data are consistent with other COVID studies suggesting that feeling isolated and lonely (Field et al., 2020c) and feeling touch deprived (Field et al., 2020b) are salient COVID-19 lockdown stress symptoms. Surprisingly, meditating was not related to those stress variables except for feeling bored. And meditating was not related to the psychological symptoms that are often comorbid with stress including anxiety, fatigue and PTSD.

Although multiple COVID-19 lockdown studies have reported stress, anxiety, and depression (Huang \& Zhao, 2020; Mazza et al., 2020; Stanton et al., 2020), as well as sleep 
disturbances (Altena et al., 2020; Cellini et al., 2020) and posttraumatic stress symptoms (Forte et al., 2020; Liu et al., 2020), none of these studies have assessed spirituality or meditation as potential buffers for these problems. In the current study, feelings of spirituality and meditating were significantly negatively correlated with these negative conditions (significant rs ranging from .14-.82). And, group differences between those who did and did not feel spiritual or did or did not meditate were also highly significant for all of the conditions. However, because of the cross-sectional nature of this study and the absence of baseline data, only relationships between variables, not effects, can be determined. These relationships may have preceded the lockdown and could have become stronger during the lockdown as coping mechanisms. Feeling spiritual and meditating were surprisingly prevalent as were the absence of worries about getting the virus and finances. And, these were accompanied by other buffering activities including connecting with friends, selftouch, self-care and exercise. Exercise was also negatively correlated with feelings of isolation and loneliness and was noted to buffer the effects of touch deprivation in a previous analysis of this database (Field et al., 2020b). Pre-COVID research has also shown positive effects of exercise on anxiety (Kandola et al., 2018) and sleep disturbances (Field, 2019). Exercise and additional touch stimulation as in hugging and back rubbing would be expected to enhance immune function via moving the skin and stimulating pressure receptors under the skin, in turn, increasing vagal activity, decreasing cortisol and increasing NK activity to ward off viral and bacterial cells (Field, 2019). Engaging in these activities may have confounded and compounded the relationships noted for spirituality and meditation. Nonetheless, those experiencing negative mood states and sleep disturbances during lockdowns might be encouraged to engage in meditation, self-touch, and exercise.

Methodologically, these data are limited by sampling and assessment issues. The sample of predominantly non-Hispanic, white females would be considered non-representative even though it may be representative of Survey Monkey samples. Accordingly, the data would not be generalizable to the larger population. In addition, the self-report data are subject to their questionable bias and reliability. Because of the cross-sectional nature of this study, and the absence of baseline data, only relationships between variables, not effects, can be determined. Future studies may compare several different groups including those feeling spiritual, those meditating, those feeling spiritual and meditating and those neither feeling spiritual nor meditating. In addition, standardized scales for spirituality and meditation may be used rather than rating scales on those variables.

Despite their limitations, these data suggest that both feeling spiritual and meditating are associated with health-related activities and reduction of sleep disturbances. And feeling spiritual in particular was associated with a reduction of stress, depression, anxiety, fatigue, and PTSD symptoms. And, neither those who reported feeling spiritual nor those engaging in meditation were experiencing the typical worries about getting the virus or about finances during this COVID-19 lockdown.

\section{References}

1. Altena, E., Baglioni, C., Espie, C. A., Ellis, J., Gavriloff, D., Holzinger, B., \& Riemann, D. (2020). Dealing with sleep problems during home confinement due to the COVID-19 outbreak: Practical recommendations from a task force of the European CBT-I Academy. Journal of Sleep Research. doiorg.fgul.idm.oclc.org/10.1111/jsr.13052

2. Anderson, M.R., Miller, L., Wickramaratne, P., Svob, C., Odgerel, Z., Zhao, R. et al.(2017).Genetic correlates of spirituality/ religion and depression: A study in offspring and grandchildren at high and low familial risk for depression. Spiritual Clinical Practices (Washington DC), 4, 43-63. Doi: 10.1037/scp0000125.

3. Braam, A.W. \& Koening, H.G. (2019). Religion, spirituality and depression in prospective studies: 
A systematic review. Journal of Affective Disorders, 257,428-438.

4. Brandmeyer, T., Delorme, A. \& Wahleh, $H$. (2019). The neuroscience of meditation: Classification, phenomenology, correlates, and mechanisms. Progress in Brain Research, 244:129. Doi: 10.1016/bs.pbr.2018.10.020. Epub 2019 Jan 16.

5. Carmody, J., Reed, G., Kriisteller, J. \& Merriam, P. (2008). Mindfulness, spirituality and healthrelated symptoms. Journal of Psychosomatic Research, 64, 393-403.

6. Cellini, N., Canale, N. Mioni, G., \& Costa, S. (2020).Changes in sleep pattern, sense of time and digital media use during COVID-19 lockdown in Italy, Journal of Sleep Research, doi. org/10.1111/jsr.13074

7. Dewitt, B., Feeny, D., Fischhoff, B., Celia, D., Hays, R.D. et al. (2018). Estimation of a preference-based summary score for the patient reported outcomes measurement information system: The PROMIS-preference (PROPr) scoring system. Medical Decision Making, 38,683-698.

8. Field, T. (2019). Social Touch, CT Touch and Massage Therapy. Developmental Review, 51,123-145.

9. Field, T., Mines, S., Poling, S., Bendell, D. \& Veazey, C. (2020a). Stress and sleep disturbances during COVID-19 lockdown. In review.

10. Field, T., Poling, S., Mines, S. Bendell, D. \& Veazey, C. (2020b).Touch deprivation and exercise during COVID-19 lockdown, Medical Research Archives, 8, 1-12.

11. Field, T., Poling, S., Mines, S., Bendell, D. \& Veazey, C. (2020c). Feeling isolated and lonely during a COVID-9 lockdown. Archives of Health Science. In press.

12. Forte, G., Faviori, F., Tambelii, R. \& Casagrande, M.(2020). COVID-19 pandemic in the Italian population: Validation of a post-traumatic stress disorder questionnaire and prevalence of PTSD symptomatology. Int. J Environ. Res. Public Health, 17, 41-51.

13. Gonzalez-Valero, G., Zurita-Ortega, F., UbagoJimenez, J.L. \& Puertas-Molero, P. (2019).Use of meditation and cognitive behavioral therapies for the treatment of stress, depression and anxiety in students. A systematic review and meta-analysis. International Journal of Environmental Research and Public Health, 16, 43-94. Doi: 10.3390/ijerph16224394.

14. Hansen, M., Anderson, T. E., Armour, C., Elklit, A., Palic,S., \& Mackrill, T. (2010). PTSD-8: A short PTSD inventory. Clinical Practice \& Epidemiology in Mental Health. 6, 101- 108.

15. Hilton, L., Maher, A., Colaiaco, B., Apaydin, E., Sorbero, M., Booth, M. et al. (2017). Meditation for posttraumatic stress: Systematic review and meta-analysis. Psychology and Trauma, 9, 453460.

16. Huang, Y. \& Zhao, N. (2020). Generalized anxiety disorder, depressive symptoms and sleep quality during COVID-19 outbreak in China: A web-based cross-sectional survey, Psychiatry Research, 288, 11-29.

17. Kandola, A. Vancampfort, D., Herring, M., Rebar, A. Hallgren. M. Firth, J. \& Stubbs, B. (2018). Moving to beat anxiety: epidemiology and therapeutic issues with physical activity for anxiety. Current Psychiatry Reports. 20: 63 doi.org/10.1007/s11920-018-0923-x

18. Knowlden, A.P., Shewmake, M.E., Burns, M. \& Harcrow, A. (2018). Sex-specific impact of spiritual beliefs and sleep quality on degree of psychological distress. Journal of Religion and Health, 57, 72-83. Doi: 10.1007/s10943-0160342-4.

19. Laird, L., Curtis, C. \& Morgan, J. (2017). Finding spirits in spirituality: What are we measuring in spirituality and health research? Journal of Religion and Health, 56, 1-20

20. Lee, D.J., Kulubya, E., Goldin, P., Gordarzi, A. \& Girgis, F. (2018). Review of the neural oscillations underlying meditation. Frontiers in Neuroscience, 12,178 Doi:10.3389/fnins.2018.00178. eCollection.

21. Liu' N., Zhang, F., Wei, C. Jia, Y. Shang, Z., Sun, L., Wu, L., Sun, Z, Zhou, Y, Wang, Y, \& Liu, W. (2020).Prevalence and predictors of PTSS during COVID-19 outbreak in China hardest-hit areas: Gender differences matter, Psychiatry Research.

22. Mazza, C., Ricci, E., Biondi, S., Coasanti, M., Ferracuti, S., Napoli, C., \& Roma, P. (2020). A nationwide survey of psychological distress among Italian people during the COVID-19 pandemic: Immediate psychological responses and associated factors. International Journal of Environmental Research and Public Health, 17, 31-65. doi:10.3390/ijerph17093165

23. Panier, L.Y.X., Bruder, G.E., Svob, C., Wickramaratne, P., Gameroff, M.J., Weissman, M.M. et al. (2020). Predicting depression symptoms in families at risk for depression: Interrelations of posterior EEG Alpha and religion/spirituality. Journal of Affective Disorders, 274,969-976. Doi: 10.1016/j.jad.2020.05.084. Epub 2020 May 27.

24. Rosmarin, D., Wackholz, A. \& Ai, A.(2000). Beyond descriptive research: advancing the study IJPRR:https://escipub.com/international-journal-of-psychological-research-and-reviews/ 10 
of spirituality and health. Journal of Behavioral Medicine, 34, 409-413.

25. Saeed, S.A., Cunningham, K. \& Bloch, R.M. (2019). Depression and anxiety disorders: Benefits of exercise, yoga, and meditation. American Family physician, 99,620-627.

26. Seeman, T., Dubin, L. \& Seeman, H. (2003). Religiosity/spirituality and Health. American Psychologist, 58, 53-63.

27. Shattuck, E.C. \& Muehlenbein, M.P. (2020). Religiousity/spirituality and physiological markers of health. Journal of Religion and Health, 59,10351054. Doi: 10.1007/s10943-018-0663-6.

28. Stanton, R., To, Q., Khalesi, S., Williams, S., Alley, S., Thwaite, T., Fenning, A. \& Vandelanotte, C. (2020). Depression, Anxiety and Stress during COVID-19: Associations with Changes in Physical Activity, Sleep, Tobacco and Alcohol Use in Australian Adults International Journal of Environmental Research and Public Health. 174065 DOI 10.3390/ijerph17114065

29. Thomas, Z., Novak, M., Torres Platas, S.G., Gautier, M., Potes-Holgin, A., Fox., R. et al.(2017). Brief mindfulness meditation for depression and anxiety symptoms in patients undergoing hemodialysis: A Pilot feasibility study. Clinical Journal of the American Society of Societal Nephrology, 12, 2008-2015. Doi: 10.2215/CJN.03900417. Epub 2017 Oct 12.

30. Tsai, P.C., Lin, J.H. \& Hsu, H.C. (2019). Exploring sleep quality, spiritual health, and related factors in nurses in the intensive care unit. Hu Li Za Zhi, 66(4): 49-59.

31. Wahleh, H. (2018). Internet Mindfulness Meditation Intervention (IMMI) improves depression symptoms in older adults. Medicines (Basel), 5,119. Doi: 10.3390/medicines5040119.

32. Yadav, R., Khanna, A. \& Singh, D. (2017). Exploration of relationship between stress and spirituality characteristics of male and female engineering students: A comprehensive study. Journal of Religion and Health, 56,388-399. Doi: 10.1007/s10943-015-0174-7.

33. Yang, E., Schambler, E., Meyer, R.M. \& Gold, J.I. (2018). Happier healers: Randomizedcontrolled trial of mobile mindfulness for stress management. Journal of Alternative and Complementary Medicine, 24,505-513. Doi: 10.1089/acm.2015.0301. Epub 2018 Feb 8. 